\title{
Desarrollo de la expresión oral como habilidad comunicativa y profesional pedagógica
}

\author{
Development of oral expression as a pedagogical communicative and \\ professional skill
}

\author{
Celeste del Valle Castillo Figueroa \\ prof.celestecastillo@gmail.com \\ Dirección y Supervisión Educativa del Ministerio de Educación
}

\section{RESUMEN}

- Artículo recibido en septiembre 2018

El propósito de la presente investigación fue proponer tareas para que el futuro educador desarrolle la expresión oral, específicamente cuando cursa el básico curricular Teoría y Práctica de la Comunicación y el Lenguaje en el Programa Nacional de Formación de Educadores (PNFE, 2006). Para ello se realizó una investigación de campo con diseño documental. La muestra fue intencional, constituida por dos profesores asesores y quince educadores en formación. Se aplicó un cuestionario a fin de evaluar los siguientes criterios: "discurso pedagógico", "fluidez verbal", "dominio del léxico" "coherencia y cohesión". Los resultados arrojaron que el primero de los indicadores estaba en el nivel "medio" y los otros tres en el "bajo", por lo que se diseñaron tareas dirigidas al favorecimiento de la expresión oral. Todo esto fue con el fin de que los educadores en formación se comuniquen oralmente de forma coherente y de esta manera se transformen en comunicadores pedagógicos.

Palabras clave: Expresión oral; discurso pedagógico; habilidad profesional pedagógica; competencia comunicativa; educadores en formación

\begin{abstract}
The purpose of this research was to propose tasks for the future educator to develop oral expression, specifically when he is studying the basic curriculum Theory and Practice of Communication and Language in the National Educators Training Program (PNFE, 2006). For this, a field investigation with documentary design was carried out. The sample was intentional, consisting of two advisory professors and fifteen educators in training. A questionnaire was applied in order to evaluate the following criteria: "pedagogical discourse", "verbal fluency", "domain of the lexicon" "coherence and cohesion". The results showed that the first of the indicators was in the "medium" level and the other three in the "low" level, so tasks designed to favor oral expression were designed. All this was in order that the educators in formation communicate orally in a coherent way and in this way they become pedagogical communicators.
\end{abstract}

Keywords: Oral expression; pedagogical discourse; pedagogical professional ability; communicative competence; educators in training 


\section{INTRODUCGIÓN}

El Programa Nacional de Formación de Educadores [PNFE] (Ministerio de Educación Superior, Ministerio de Educación y Deportes y Universidad Bolivariana de Venezuela, 2006) tiene como reto fundamental priorizar la optimización del proceso docenteeducativo y con ello la enseñanza de una lengua materna que favorezca en los estudiantes el desarrollo de sus destrezas básicas, en correspondencia con sus necesidades, intereses y motivaciones.

De allí que fue estimado como universo investigativo la necesidad de reorientar la enseñanza de la lengua, a partir de perspectivas más actualizadas como son el enfoque comunicativo y la lingüística textual. De esta manera, el docente podría considerar situaciones reales de comunicación para que nuestros estudiantes logren "aprender a usar, crear y recrear la lengua” (Hernández, 2011); además, de que también se favorece el desarrollo y práctica de estrategias cognitivas y metacognitivas, las cuales redundarán en beneficio de sus capacidades comunicativas $y$, por ende, del uso adecuado de la lengua.

Desde las posiciones del lingüista Hymes (1971) hasta las teorías de van Dijk (1984), múltiples investigaciones coinciden en reconocer que hay una estructura en el uso del lenguaje que va más allá de los aspectos contemplados en la gramática. Este nuevo enfoque, surgido de la confluencia de la sociolingüística, la filosofía del lenguaje, la psicolingüística, de la lingüística del texto, entre otras, tiende al establecimiento de una teoría que incluye reglas sintácticas, también reglas semánticas y pragmáticas, cada una de ellas distinta en su forma y en su función y que trata de explicar la naturaleza social del lenguaje y su realización en el proceso de interacción de los hombres en un contexto social determinado.

De estos presupuestos surge una de las nociones seminales para la enseñanza de la lengua materna y el enfoque comunicativo del lenguaje: el de competencia comunicativa, que tiene en cuenta no solo la realización concreta del acto de habla sino también el contexto en que se produce ese acto y los diversos espacios en los cuales se lleva a cabo, como por ejemplo el universitario (Benoit, 2018).

En consecuencia, la noción de competencia comunicativa ha contribuido a precisar los fines de la enseñanza de la lengua materna: en los primeros niveles el niño trae el saber intuitivo sobre su lengua, un conocimiento general de la estructura de su lengua, pero acarrea numerosas fallas en cuanto a su competencia comunicativa. $\mathrm{Y}$ es al desarrollo de esta competencia tanto en forma oral como escrita a la que debemos atender. 
Para Hymes (1971) la competencia comunicativa permite que el individuo sea autónomo en cuanto al uso del lenguaje, ya que será este el que decida momento, tema, forma, dónde y cuándo hablar, además de que podrá ser capaz de evaluar cómo fue su actuación y la de los otros.

De ahí que la metodología de la enseñanza deba propiciar el desarrollo de las habilidades comunicativas a partir de la aplicación en el área docente de procedimientos y modelos de análisis textual que enseñen a comunicar, atendiendo a reglas lingüísticas $y$ también a las reglas socioculturales que rigen la interacción verbal y de aprendizajes significativos, que aseguren la continuidad del conocimiento aprendido durante los procesos de enseñanza y aprendizaje, su interrelación con el conocimiento previo que el estudiante posee y la aplicación de este con un fin utilitario: significa entonces, sentar las bases de una enseñanza desarrolladora y convertir en herramienta de trabajo el mejor instrumento de comunicación humana (Echevarría, Roque y Pérez, 2013).

Uno de los componentes de la enseñanza de la lengua materna que debe atender a esta visión integradora y que contribuye al desarrollo pleno de la competencia comunicativa, es el relacionado con la expresión oral por cuanto es una herramienta ineludible en los procesos de simbolización, de construcción del conocimiento y para el desempeño profesional del docente en formación.

Siguiendo el hilo conductor del desarrollo de la habilidad comunicativa se decidió realizar un diagnóstico a los educadores en formación de la aldea Universitaria "Mireya Vanegas”, el cual reveló como elementos más significativos, la existencia de limitaciones en la coherencia, cohesión y concordancia en el discurso oral de algunos educadores en formación. Por otro lado, se manifestaron insuficiencias en el grado de eficacia de la metodología puesta en práctica, en la orientación metodológica-conceptual que la sustenta y en el nivel de sistematización del texto oral para la organización y materialización de juicios claros, así como su incidencia en la futura actividad profesional del educador en formación. Los contenidos referentes a la comunicación no han logrado profesionalizarse de modo que revelen su utilidad para el ejercicio de la profesión del futuro educador.

Todo lo anterior revela la existencia del problema científico siguiente: ¿De qué manera favorecer al desarrollo de la expresión oral como habilidad comunicativa profesional pedagógica en los docentes en formación del PNFE Aldea Universitaria “Mireya Vanegas”? 
La investigación precisó que este problema se manifestara en los procesos de enseñanza y aprendizaje del básico curricular Teoría y Práctica de la Comunicación y el Lenguaje. Ello derivó en la determinación del objetivo: proponer tareas docentes para el desarrollo de la expresión oral como habilidad profesional pedagógica en los educadores en formación del PNFE Aldea Universitaria "Mireya Vanegas”, con lo que se precisó como campo de acción el desarrollo de la expresión oral como habilidad comunicativa y como habilidad profesional pedagógica.

Una habilidad profesional que debe ser desarrollada desde los primeros estadios de la formación del futuro profesional de la educación es la expresión oral. Esta es la habilidad lingüística relacionada con la producción del discurso oral. Es una habilidad comunicativa que abarca no solo un dominio de la pronunciación, del léxico y la gramática, sino también conocimientos socioculturales y pragmáticos. Es una habilidad totalmente productiva que al decir de Roméu (2005) es entendida como un saber y hacer en situaciones sociales comunicativas, que exige de los participantes en la comunicación capacidades para adecuar la significación y los medios que se usan para significar a las exigencias del contexto, las jerarquías de los participantes, las relaciones de poder $y$ otros factores.
Lo anterior evidencia las dimensiones de la competencia comunicativa: los procesos cognitivos implicados en la significación, las estructuras discursivas y el modelo de contexto que el individuo se ha construido y que lo lleva a adecuar la comunicación a la situación social comunicativa, donde se revelan sus conocimientos, intenciones, sentimientos, ideología y jerarquía social. Esto permite constituir lo que para van Dijk (1984) es el "triángulo del discurso”, conformado por tres componentes: cognición, discurso y sociedad. Cada uno depende de los demás, es imposible que funcionen por separado.

Desde esta dinámica, el ser competente para construir significados implica ser un comunicador eficiente, que se caracterice por su capacidad para integrar el saber (conocer), el saber sery el saber convivir (componente afectivomotivacional y recursos personológicos y axiológicos) y el saber hacer (desenvolvimiento en el acto de comunicación como ser social) para poder expresarse con creatividad en cada contexto de actuación profesional.

En las últimas décadas investigadores tales como Fernánde $z$ (2002), Forgas (2003), Parra (2004) han connotado la transversalidad de la competencia comunicativa, así como la importancia de su desarrollo en el educador. Por otra parte, esta competencia ha sido 
relanzada desde lo teórico como un factor de la eficiencia profesional, aspecto que dimensiona su matiz profesional pedagógico y que trasciende propiamente lo lingüístico.

Desde un enfoque pedagógico Ortiz (1997) concibe la competencia comunicativa como "la capacidad del maestro para establecer una comunicación efectiva y eficientemente óptima (con sus alumnos), al desarrollar en su personalidad un estilo de comunicación flexible y lograr resultados educativos deseados" (s.n). Desde la perspectiva de la autora, el desarrollo de la expresión oral que se perfila hacia el logro de la competencia comunicativa constituye un modo de actuación y un problema profesional que debe solucionar el educador en formación con respecto al objeto de la profesión, es una de las exigencias a la personalidad del maestro para ser competente comunicativamente e influir positivamente en el desarrollo de estas en los educandos.

Finalmente, se asume que la asunción de la competencia comunicativa en los procesos de enseñanza y aprendizaje de Teoría y Práctica de Comunicación y lenguaje con un sentido profesional implica el desarrollo de cada una de las habilidades lingüísticas y de forma especial de la expresión oral.

El programa del básico curricular Teoría y Práctica de la Comunicación y el Lenguaje del componente Formación Socio Cultural, que se toma como objeto de análisis tiene como propósito facilitar la apropiación, el uso y perfeccionamiento de las formas de lenguaje y estrategias comunicacionales de los educadores en formación del Programa Nacional de Formación de Educadores. Sin embargo, desde la perspectiva de la autora de esta investigación, el objetivo planteado no ha sido matizado de la necesaria profesionalización que deben tener los contenidos curriculares en función del ejercicio de la profesión del educador en formación. Desde su planteamiento, el objetivo revela limitaciones en relación con los problemas profesionales que el educador en formación tendrá que enfrentar y resolver con el uso adecuado de la lengua materna. En el Cuadro 1 se presentan las sugerencias para la distribución del sistema de contenidos, subrayando los contenidos a los cuales se les dio tratamiento en el diseño de las tareas docentes propuestas. 
Guadro 1. Sugerencias para la distribución de contenidos del básico curricular Teoría y Práctica de la Comunicación y el Lenguaje

\begin{tabular}{ll}
\hline & Segundo trayecto de formación intermedia \\
\hline Segundo semestre & Duración: 60 Horas \\
\hline Contenidos & Tiempo/ horas \\
\hline
\end{tabular}

III La lengua oral:

Reflexiones sobre la lengua oral:

- La petición más frecuente en la escuela: ¡Silencio!

- La lengua oral en el contexto educativo.

- Variedades lingüísticas.

- La tradición oral de nuestro pueblo.

Estrategias para expresión oral:

20 horas

- Habilidades lingüísticas:

-Comprensión oral

-Expresión oral.

IV La lengua gestual:

- Lo no verbal en la oralidad.

- La expresión corporal.

La lengua de señas (artículo 81 de la CRBV)

V La comunicación:

- La comunicación interpersonal:

- El proceso de la comunicación.

- Filtros culturales, fisiológicos y Psicológicos.

- La interpretación y la comunicación.

- La comunicación intrapersonal.

- El problema de la "comunicación objetal"

20 horas

- La comunicación no violenta.

- El discurso en el aula.

- La comunicación con los padres y representantes.

- La comunicación entre pares.

- La comunicación entre los (las) diferentes actores.

- Las redes de comunicación.

Fuente: Programa Nacional de Formación de Educadores y Educadoras. Ministerio de Educación Superior, Ministerio de Educación y Deportes y Universidad Bolivariana de Venezuela (2006). 
Reflexiones sobre la comunicación en el ámbito escolar

Se pondera que los diferentes temas tienen delineados sus objetivos. Sin embargo las habilidades no reflejan una aproximación ascendente a los contenidos en tanto no siempre se presentan de manera escalonada de lo fácil a lo difícil, de lo simple a lo complejo, y que debe ser expresión del tránsito por los distintos niveles de asimilación y de desempeño, cuestión que evidencia limitaciones en el microdiseño curricular.

De esta manera, al analizar el sistema de contenidos del básico curricular señalado, se percibe que quedan explícitos los conocimientos que el educador en formación tendrá que aprehender, estos como aquellos rasgos del contenido refractados en la conciencia del mismo. De la misma forma se presentan las habilidades. Sin embargo, las mismas no están orientadas, desde el microdiseño curricular, para convertirse en modos de actuación para el educador en formación. Lo anterior permite plantear que el educador en formación no es concientizado de la significación social que tienen tales contenidos para el ejercicio de la profesión ni lo pone en contacto con el objeto de la profesión desde este básico curricular.
Por otro lado, en el básico curricular no se refieren aquellos valores que son el resultado de la interactuación entre el docente en formación y el objeto de estudio de Teoría y Práctica de Comunicación y Lenguaje. En tal sentido, el docente en formación no concientiza en toda su plenitud el significado social de ese objeto.

Se refleja una carencia en la especificación de los métodos particulares de esa didáctica para poder conducir los procesos de enseñanza y aprendizaje; las orientaciones metodológicas no expresan la semántica del término y se le limitan a ofrecer técnicas que, a criterio de la autora de esta investigación, se sustentan en determinados métodos y enfoques que rigen la enseñanza de la lengua materna en la actualidad. No obstante, desde el microdiseño curricular del básico Teoría y Práctica de Comunicación y Lenguaje no se potencian la interdisciplinariedad que permite la solución de los problemas profesionales que el estudiante debe resolver en su formación desde la integración de los contenidos de la profesión donde la expresión oral se revela como una habilidad profesional pedagógica.

\section{MÉTODO}

La base metodológica que orientó la presente investigación fue la dialécticamaterialista y los métodos de 
investigación utilizados fueron los siguientes:

\section{Del nivel teórico:}

Análisis y síntesis: se descompuso en sus determinaciones sustantivas el básico curricular de Teoría y práctica de la comunicación y el lenguaje y de la expresión oral.

Inductivo-deductivo: se analizaron los procesos de enseñanza y aprendizaje del básico curricular Teoría y práctica de la comunicación y el lenguaje y de la expresión oral como totalidades desde lo particular hasta lo general.

\section{Del nivel empírico:}

Análisis documental: se utilizó en la revisión, análisis e interpretación del programa del básico curricular Teoría y práctica de la comunicación y el lenguaje, así como en la revelación de sus fortalezas y debilidades.

Observación a clases: para evidenciar la problemática existente con relación a la expresión oral así como la profesionalización de la misma en el proceso de enseñanza-aprendizaje en el básico curricular Teoría y Práctica de la Comunicación y el Lenguaje.

Consulta a especialistas: se validó la propuesta de las tareas diseñadas con los
15 especialistas quienes dieron sus criterios y opiniones.

Entrevista: se evidenció por medio de los 2 profesores asesores del PNFE que imparten el básico curricular Teoría y práctica de la comunicación y el lenguaje, el estado de opinión y dominio teórico- metodológico sobre la expresión oral.

Métodos matemático estadísticos: se utilizó la estadística descriptiva que permitió describir lo realizado en el diagnóstico y la valoración, el análisis porcentual expresado en tablas $\mathrm{y}$ gráficas para evaluar cualitativamente y cuantitativamente cada instrumento aplicado y los resultados obtenidos.

La población estuvo conformada por 2 profesoras asesoras y dos secciones conformadas por 30 educadores en formación del PNFE Aldea Universitaria "Mireya Vanegas".

La muestra se constituyó por 2 profesoras asesoras y una sección de 15 educadores en formación del PNFE Aldea Universitaria “Mireya Vanegas”. El criterio de inclusión atendió a las siguientes especificaciones: a) pertenecer al Programa Nacional de Formación de Educadores y Educadoras, y b) realizar vinculación profesional y cursar el segundo semestre. En tal sentido, la población objeto de estudio es de tipo finito. 
La prueba pedagógica aplicada a los educadores en formación del segundo semestre de educación de la Aldea Universitaria "Mireya Vanegas", se realizó con un cuestionario dirigido a valorar el comportamiento de las habilidades que conforman la expresión oral. Son estas: "discurso pedagógico", "coherencia y cohesión”, "fluidez verbal" y "dominio del léxico". A cada indicador se le asignaron medidas de valor evaluadas en Muy alto (MA), Alto (A), Medio (M), Bajo (B). Después de aplicados los instrumentos se realizó el procesamiento de la información, el cual permitió determinar el estado de los educadores en formación.

\section{RESULTADOS}

En el primer indicador denominado Discurso pedagógico, cinco obtuvieron "Bajo" para un 33,3 \%, por no saber expresarse, nueve la evaluación de "Medio" que representa el $60 \%$, ya que demostraron poseer claridad de ideas y uno recibió la evaluación de "Alto" que representa un 6,6\%, al expresar con claridad las ideas.

En el segundo indicador, Coherencia y cohesión en su discurso, nueve no fueron coherentes, se evaluaron de "Bajo" y representa el $60 \%$, uno fue evaluado de "Medio" por presentar algunos problemas de coherencia en su lenguaje proyectó un 6,6\%, y cinco fueron evaluado de "Alto" que representa el 33,3\%, por presentar un lenguaje coherente.

En el tercer indicador se midió la Fluidez verbal, diez obtuvieron "Bajo" para un $66,6 \%$, tres obtienen la categoría de "Medio" que representa un $20 \%$ porque utilizaron entonación en su lenguaje aunque faltó fluidez, dos se evaluados de “Alto" y representa el 13,3\%, ya que presentaron fluidez y entonación en su lenguaje.

En el cuarto indicador, Dominio del léxico, la utilización adecuada del vocabulario, nueve obtuvieron "Bajo" para un $60 \%$ por no presentar un vocabulario adecuado, tres obtuvieron "Medio" para un 26,6 \% por presentar un vocabulario pobre, y dos fueron evaluados de "Alto" y representa el 13,3 $\%$, que si utilizaban correctamente las palabras de acuerdo a su significado.

Sobre la base de los resultados antes descritos, fueron diseñadas nueve tareas para el desarrollo del básico curricular Teoría y Práctica de Comunicación y Lenguaje. Estas tareas presentaron las siguientes cualidades: a) flexibles $y$ dinámicas; b) diversas; c) acordes con las necesidades de los educadores en formación; d) inversión de un mínimo de recursos; e) carácter participativo; f) transdisciplinares; g) creativas. A continuación se presentan las tareas docentes, según la estructura asumida. 


\section{Tarea 1. De la escucha a la oralidad:}

- Tema: La lengua oral

- Objetivo: analizar la coherencia y cohesión oral para la adecuada comprensión auditiva en situaciones comunicativas.

- Contenido: comprensión auditiva (coherencia y cohesión)

Situación de aprendizaje: análisis de canción

Audición de la canción "El cantar tiene sentido” (polo margariteño) perteneciente al folklore venezolano interpretado por Soledad Bravo, la cual tiene la transcripción incompleta.

- Inciso A: forme parejas de trabajo para luego de escuchar la letra complete la misma.

- Inciso B: analice con su pareja el contenido de la letra elaborada.

- Inciso $C_{:}$exponga uno de los miembros de la pareja la pieza musical, para ello improvise desde la comprensión realizada de la misma.

- Método: elaboración conjunta

- Formas de organización: clase práctica

- Medios de enseñanza: dispositivo para reproducir audio

- Evaluación: autoevaluación y coevaluación.

\section{Tarea 2. Colectivo oral:}

- Tema: Reflexión sobre la comunicación en el ámbito escolar

- Objetivo: argumentar el papel de la coherencia y cohesión para la adecuación del discurso oral pedagógico en ámbitos educativos.

- Contenido: el discurso en el aula (Coherencia y cohesión)

\section{Situación de aprendizaje:}

Elabore un cuento en forma narrativa de una experiencia pedagógica de su vinculación profesional en la cual participen varios elementos según las siguientes imágenes correspondientes a tres componentes que deben integrarse y representarse en el cuento.

- Lugares: la dirección del colegio, una biblioteca, un aula, el patio del colegio, Consejo de protección del niño y del adolescente.

- Objetos: una pizarra, una libreta, un formato de entrevista con el representante, registro descriptivo, una carta de amor.

- Personajes. un director, un representante, un estudiante de primaria, una trabajadora social, un docente de aula.

- Inciso A: forme equipos de trabajo de cinco integrantes y emplee para relatar la historia la técnica "cuenta cuento".

- Inciso B: argumente la narración en la clase práctica. 
- Método: elaboración conjunta

- Formas de organización: clase práctica

- Medios de enseñanza: lápiz, páginas blancas, imágenes de las categorías

- Evaluación: autoevaluación y coevaluación.

\section{Tarea 3. El orador}

- Tema: la comunicación

- Objetivo: argumentar ideas para la adecuación del discurso pedagógico en ámbitos formales.

- Contenido: la comunicación entre diferentes actores (discurso pedagógico)

\section{Situación de aprendizaje:}

A partir de la lectura del periódico Líneas de Chávez en el artículo "Llevo tu luz y tu aroma en mi piel..."

- Inciso A: elabora un artículo de opinión, en el cual, con el empleo de la técnica "la emisora de radio", reporte la noticia desde su perspectiva pedagógica.

- Inciso B: argumente en colectivo la calidad del artículo elaborado.

- Método: elaboración conjunta y trabajo independiente

- Formas de organización: taller

- Medios de enseñanza: periódico, páginas blancas

- Evaluación: autoevaluación y coevaluación.

\section{Tarea 4. Expresando emociones}

- Tema: la comunicación

- Objetivo: caracterizar el discurso oral a través de sus componentes para la adecuada interpretación comunicativa.

- Contenido: la comunicación interpersonal (fluidez verbal)

\section{Situación de aprendizaje:}

A partir de la visualización de la película "Hermano" director Marcel Rasquin.

- Inciso A: escenifique en equipos de trabajo de cinco integrantes la escena que más le haya cautivado, creando según recuerde los parlamentos de los mismos.

- Inciso B: caractericé cuáles son las costumbres y tradiciones contenidas en esa escena.

- Método: trabajo independiente

- Formas de organización: dramatización

- Medios de enseñanza: vestuario, máscaras, escenografía, dispositivo reproductor de video

- Evaluación: autoevaluación y coevaluación

Tarea 5. Expresión en la interacción tecnológica

- Tema: reflexión sobre la comunicación en el ámbito escolar 
- Objetivo: Exponer criterios y Tarea 6. Estudio de caso opiniones a través del dominio del léxico para las distintas fuentes de discusión oral, promoviendo el aprendizaje en lo estudiantes.

- Contenido: las redes de comunicación (dominio del léxico)

Situación de aprendizaje: análisis de opiniones en el blog educativo

Analice las opiniones contenidas en el blog educativo "Edúcame Educando" además de colgar un comentario debidamente fundamentado sobre el tema las "habilidades profesionales", desde una perspectiva pedagógica. Elabóralo en equipos de cinco integrantes.

- Inciso A: la publicación de su comentario deberá realizarla en un periodo de siete días, para que sus compañeros de clase puedan leerlo e interactuar.

- Inciso B: exponer sus comentarios que deberán imprimirse para ser socializados en el aula de clase, ajustándose a las exigencias de este tipo de comunicación colectiva.

- Formas de organización: taller

- Medios de enseñanza: computador, internet, páginas blancas

- Evaluación: autoevaluación y coevaluación.

- Tema: reflexión sobre la comunicación en el ámbito escolar

- Objetivo: Caracterizar un estudiante de la vinculación profesional mediante un estudio de caso para la correcta interacción pedagógica en situaciones comunicativas educativas.

- Contenido: comunicación con los padres y representantes (interacción pedagógica).

Situación de aprendizaje: observación de la actividad verbal del niño en el contexto escolar.

Auxiliado en los métodos y técnicas de investigación empíricas, realice un estudio de caso con la ayuda de su profesor tutor, para lo cual debe desarrollar los pasos siguientes:

- Inciso A: observación de la actividad del niño en el contexto escolar.

- Inciso B: prepárese para caracterizar los resultados alcanzados en el estudio de caso en la clase.

- Método: trabajo independiente, elaboración conjunta

- Formas de organización: taller

- Medios de enseñanza: presentación digital

- Evaluación: autoevaluación y coevaluación. 


\section{Tarea 7. Comprender para relatar:}

- Tema: la comunicación

- Objetivo: Analizar la efectividad de la coherencia y cohesión promoviendo su aprendizaje a través de formas de interacción pedagógica distribuidas en espacios y tiempos diferentes.

- Contenido: el proceso de comunicación (coherencia y cohesión).

\section{Situación de aprendizaje:}

Diseño de una tarea de aprendizaje de comprensión oral para los estudiantes en su vinculación profesional.

Realice un formato de entrevista para ser aplicada al profesor tutor sobre su actividad pedagógica.

- Inciso A: elabore el cuestionario.

- Inciso B: realice la entrevista.

- Inciso $C$ : elabore un informe para presentarlo oralmente en la clase.

- Inciso D: analice el papel de los actores en el proceso de comunicación en ámbito escolar.

- Método: trabajo independiente, elaboración conjunta

- Formas de organización: clase práctica o taller

- Medios de enseñanza: televisor

- Evaluación: autoevaluación y coevaluación.

\section{Tarea 8. Grafismo verbal}

- Tema: la lengua oral

- Objetivo: exponer el problema didáctico de la integración de los contenidos de la expresión oral con los de la profesión a partir de la interacción pedagógica promoviendo su aprendizaje.

- Contenido: expresión oral (interacción pedagógica sus componentes. Funciones. Barreras de la comunicación)

\section{Situación de aprendizaje:}

Oriente a sus estudiantes en su vinculación profesional, elaborar un medio de enseñanza gráfico donde utilicen la imaginación, que esté ajustado al contexto escolar.

- Inciso A: ¿qué orientaciones precisa usted, le dará al estudiante?

- Inciso B: argumente los aspectos a evaluar en dicha actividad.

- Inciso $C_{\text {: }}$ prepárese para exponer sobre la tarea en clase.

- Método: trabajo independiente

- Formas de organización: clase práctica

- Medios de enseñanza: creyones, lápiz, páginas blancas

- Evaluación: autoevaluación y coevaluación 


\section{Tarea 9. Olimpíada de la expresión} oral

- Tema: la lengua oral

- Objetivo: valorar la efectividad de la expresión oral para la correcta interacción pedagógica en situaciones comunicativas educativas.

- Contenido: expresión oral (Interacción pedagógica sus componentes. Funciones. Barreras de la comunicación)

\section{Situación de aprendizaje:}

Promueva entre los estudiantes con los cuales usted interactúa durante la clase un concurso de décimas alegóricas a la Cruz de mayo, utilizando el estilo de expresión del contrapunteo.

- Inciso A: seleccione las vías para divulgar el concurso.

- Inciso B: proponga los parámetros para la inclusión de los participantes y su evaluación.

- Inciso $C$ : presentación de los concursantes en la ejecución del contrapunteo.

- Inciso D: valore los resultados obtenidos de la ejecución.

- Método: trabajo independiente, elaboración conjunta.

- Formas de organización: taller

- Medios de enseñanza: hojas de trabajo, computador
- Evaluación: autoevaluación y coevaluación.

Dentro de las características vinculadas con las tareas docentes, la integración de habilidades es muy importante, también la dinámica grupal, sin dejar de hacer referencia a las técnicas participativas como elementos motivacionales. La propuesta asumida por la autora garantiza el proceso de socialización, prepara al educador en formación para una mejor comunicación en el medio social donde se desarrolla y propicia, mediante acciones y aptitudes concretas asumidas a través de las tareas docentes, el mejoramiento del desarrollo de la expresión oral como habilidad profesional pedagógica.

En tal sentido, los datos reales derivados de los instrumentos aplicados revelan el carácter objetivo de las insuficiencias respecto a la expresión oral en los procesos de enseñanza y aprendizaje de Teoría y Práctica de Comunicación y Lenguaje, las que limitan el desempeño eficiente del educador en formación en el contexto laboral. Los datos teóricos y empíricos connotan las insuficiencias con respecto a la expresión oral como habilidad profesional pedagógica, elementos que demuestran la necesidad y pertinencia de aportar una solución en este orden. 
Constatación de la factibilidad las de tareas docentes propuestas

Para darle una valoración objetiva a la propuesta se recurrió a la consulta de especialistas, tomando en consideración la importancia de las fuentes de argumentación basadas en su experiencia y formación teórica. Con este propósito se seleccionaron 20 especialistas que ostentan el grado académico de doctor en educación, especialista en estrategias de aprendizaje, profesor universitario de castellano y literatura con 23 años de servicio.

En la primera vuelta fue posible determinar algunas de las limitaciones que presentaba la propuesta, se valoró por parte de los especialistas mejorar la redacción de las tareas docentes, las cuales contribuyeron a perfeccionar y enriquecer la propuesta realizada.

En una segunda ronda, se recibieron las veinte respuestas para un $100 \%$ que después de procesadas arrojaron los siguientes resultados:

Como se puede apreciar del total de respuestas posibles (240), se obtuvo una valoración de Muy Apropiado en un total de 229 respuestas, para un $93.45 \%$ y el resto de respuestas posibles (11) fueron en la categoría de Bastante Apropiado, para un $6.54 \%$.
También se debe destacar que los criterios relativos a pertinencia de las tareas docentes, posibilidad de aplicación en la práctica social, recibieron la categoría de Muy Apropiado, de la totalidad de los especialistas encuestados.

En cambio, los criterios referidos a estructura de las tareas docentes, metodología de las tareas docentes, uso del lenguaje científico en las tareas docentes y coherencia y lógica de la exposición escrita de las ideas, fueron los que recibieron la menor aceptación en la categoría de Muy Apropiado (12 de 20) y los ocho restantes los evaluaron de Bastante Apropiado.

Además de los elementos anteriores, en la consulta a especialistas se alcanzaron los siguientes criterios:

$\checkmark$ "Me parece muy oportuna"

$\checkmark$ "Ojalá las actividades se generalicen"

$\checkmark$ "Hacía mucha falta este tipo de propuesta"

$\checkmark$ "Estoy de acuerdo con la propuesta"

$\checkmark$ "Para su implementación es necesario la preparación de los docentes"

En el procesamiento estadístico de la consulta realizada se evidenció un consenso de los especialistas que conduce a considerar como Muy Apropiada la propuesta; no obstante las sugerencias y los señalamientos realizados, así como los criterios 
emitidos por los mismos en intercambios con la investigadora, permitió realizar cambios y modificaciones en algunos aspectos relacionados con la propuesta.

\section{Análisis de los resultados de la aplicación de las tareas docentes}

Para comprobar la efectividad de la propuesta luego de su aplicación se emplearon métodos de investigación empíricos en los que se obtuvieron los siguientes resultados.

Como parte del diagnóstico aplicado una vez puesta en práctica la propuesta se realizó la observación de tres clases y se comprobó que:

La autora considera que los resultados de las tareas docentes aplicadas a los educadores en formación de la muestra seleccionada, satisface la expectativa, al evidenciar un nivel de motivación hacia el básico curricular Teoría y Práctica de la Comunicación y el Lenguaje, al desarrollo de habilidades profesionales pedagógicas y en especial a las actividades de expresión oral, por lo que es obvia su aceptación. En cuanto a las tareas desarrolladas contaron con creatividad y resultados interesantes.

El $100 \%$ planteó razones que argumentaron la importancia de la expresión oral en la labor docente, manifestando que interiorizan lo excepcional de este trabajo para el desenvolvimiento con éxito en la vida práctica, presente y como futuros educadores.

\section{CONCLUSIONES}

Durante los procesos de enseñanza y aprendizaje del básico curricular Teoría y Práctica de la Comunicación y el Lenguaje se debe concebir el desarrollo de la expresión oral como habilidad profesional pedagógica como un proceso organizado, planificado, ejecutado $\mathrm{y}$ evaluado de manera integral, flexible, sistemático y profesionalizado, a partir de promover un aprendizaje desarrollador, sustentado en los principios didácticos y el enfoque histórico cultural.

De esta manera, la existencia de insuficiencias en el desarrollo de la expresión oral como habilidad profesional pedagógica en los estudiantes del segundo semestre de la Aldea Universitaria "Mireya Vanegas" restringe el cumplimiento de los objetivos formativos del PNFE y en particular del básico curricular Teoría y Práctica de la Comunicación y el Lenguaje al constatarse limitaciones en la coherencia, cohesión y concordancia en el discurso de estos durante el acto pedagógico.

Por esta razón, la aplicación y análisis de los diferentes instrumentos de la investigación permitió constatar los 
problemas existentes en el desarrollo de la expresión oral como habilidad profesional pedagógica de los educadores en formación de segundo semestre de la Aldea Universitaria "Mireya Vanegas" y sentar las bases para elaborar las tareas docentes. Las principales dificultades detectadas fueron: escasez de vocabulario, temor $y$ limitado desarrollo de la expresión oral, falta de fluidez verbal y entonación, entre otras.

Las tareas docentes se caracterizan por ser un medio eficaz que interacciona con los educadores en formación en su preparación. Es desarrollador, axiológico, gradual y socializador; de manera tal que los educadores en formación logran desarrollar progresivamente la expresión oral como habilidad profesional pedagógica, y contribuye a su formación integral, objetivo fundamental de la educación del país y de la sociedad.

Finalmente, con la aplicación de las tareas docentes, se contribuyó al desarrollo de la expresión oral como habilidad profesional pedagógica en los educadores en formación de la Aldea Universitaria "Mireya Vanegas", ubicada en Caricuao, Caracas, porque lograron poseer sus propias normas y recursos expresivos, en filiación de la situación comunicativa que se les presente.

\section{REFERENCIAS}

Benoit R., C. (2018). Competencia comunicativa en una actividad curricular de lenguaje con estudiantes chilenos de Pedagogía. Revista Espacios, 39(46). Recuperado de https://revistaespacios.com/a18v 39n46/a18v39n46p24.pdf

Echevarría B., N., Roque C., K. y Pérez B., M. (2013). La comunicación oral pedagógica como habilidad. Revista Mendive Científico Pedagógica, 11(44), 1-5. Recuperado de https://dialnet.unirioja.es https://dialnet.unirioja.es

Fernández, G. (2002). Habilidades para la comunicación y la competencia comunicativa. En: Fernández G AM. Comunicación Educativa. 2da ed. La Habana: Pueblo y Educación

Forgas, B. (2003). Diseño curricular por competencias: Una alternativa para la formación de un técnico competente. ISP "Frank País". Colección Pedagogía 2003. La Habana: Palacio de las Convenciones Hernández L., V. M. (2011). Razones didácticas para fundamentar la expresión escrita, como proceso de competencia y actuación. Integra Educativa, 4(1), 205-218. Recuperado de http://www.scielo.org.bo/pdf/rieiii/ v4n1/v4n1a12.pdf

Hymes, D. (1971). Foundations in sociolinguistics: an ethnographic approach, Volume 6 (Reprinted in 2001 by Routledge) London: International Journal of Cross Cultural 
Ministerio de Educación Superior [MES], Ministerio de Educación y Deportes [MED] y Universidad Bolivariana de Venezuela [UBV] (2006). Programa Nacional de Formación de Educadores y Educadoras. Comisión Nacional-PNFE. Caracas, Venezuela. Recuperado de https://es.calameo. com/read/000233168bf80207797f8

Ortiz, E. (1997). ¿Cómo desarrollar la competencia comunicativa del maestro? Colección Pedagogía 97. La Habana: Palacio de las Convenciones

Parra, R. (2004). La competencia comunicativa profesional pedagógica: una aproximación a su definición. Recuperado de http://www.google.com/competencia 2004
Roméu, A. (2005). Propuesta didáctica basada en el Enfoque Cognitivo, Comunicativo y Sociocultural para la Enseñanza del Discurso como Objeto Complejo. Instituto Superior Pedagógico "Enrique José Varona" VARONA. Revista CientíficoMetodológica. Recuperado dehttp://www.eumed.net/libros/2011a /922/enfoque\%20cognitivo\%20comu nicativo $\% 20$ y $\% 20$ sociocultural $\% 20 \mathrm{e}$ n\%20la\%20ensenanza\%20de $\% 201 a \%$ 20Lengua\%20Materna.htm

Van Dijk, T.A. (1984). Prejudice in discourse an analysis of ethnic prejudice in cognition and conversation. Amsterdam Philadelphia: J. Benjamins Co 